\title{
A CONTRIBUTION TO THE TRAFFIC STATE ESTIMATION BY MEANS OF IMAGE PROCESSING
}

The success of traffic simulations depends largely on the simulation model validity and on accuracy of input data. For input data acquisition, video cameras are used to survey the traffic at junctions or at other places along the road to collect video data. The velocity, distance between the vehicles, acceleration and other relevant parameters can be extracted from the collected data. The estimated values are used for calibration of the simulation model. The data that has to be collected for calibration or validation of the simulation system, differ from country to country and reflects the driver behavior. For that reason, the data collection must be performed and the simulation system calibrated, each time when modeling a different traffic area. The more accurate the obtained parameters, the more accurate the traffic simulation would be. The image processing methods and the expected accuracy of traffic state estimation, such as vehicle position, speed and acceleration, is discussed in the paper and applied to the evaluation of driver behavior necessary for the calibration of the microscopic simulation systems.

Keywords: microscopic systems, transportation simulation, error estimation, image processing

\section{Introduction}

There are many areas of traffic applications where the estimation of traffic states is necessary. A variety of methods can be applied depending on the environment, i.e. the objects in the scene and many other factors. This paper concentrates on the estimation of traffic states necessary for calibration of the microscopic simulation models. Such models describe vehicle dynamics in detail and can be used to evaluate future impacts of projected new road constructions or changes in traffic control. Furthermore, the traffic states of interest are position of the vehicle, its velocity and acceleration. Also in this subfield, there are many possibilities how to obtain the mentioned traffic state values. The important characteristic of the estimated traffic states is the acquired data precision. There are not many known results on defining the methodology which investigates all possible error sources during the process of traffic state evaluation. The main goal of this paper is to contribute to the methodology for the error estimation. The traffic applications that can profit from this work are the traffic simulation systems which have to be calibrated and this calibration has to be validated.

The second chapter describes the car following model. This model is used by the majority of the traffic simulation systems. The third chapter describes the common framework structure for vision surveillance systems which is used to collect the data from the scene. The error sources are identified and the proposal how to calculate and validate them. The chapters four and five describe the calculation of the position and velocity and the calibration of the system, respectively. The final chapter is the conclusion and the outline of the future work.

\section{Car Following Models}

Microscopic traffic flow models simulate the behavior of single traffic participants. The dynamic variables of the model for the simulated vehicles are the position and the velocity of the single vehicle. There are many models used in traffic simulation which basically belong to two major groups: car-following models and cellular automation models. For this work the model of interest was the car-following model.

Car following models are also known as time-continuous models, since the transportation flow is modeled as a continuous not a discrete process.

The car following process is depicted in Fig. 1, [1]. The vehicles are considered inertial systems. All vehicles are modeled with the same average characteristics: the average length $L_{V}$, the average speed $v$ and the average distance $L$ from the previous vehicle. The parameter $L_{S}$ is the minimum safety distance between the vehicles. All vehicles are modeled with the same average parameters.

The basic characteristics of the transportation flow are:

$v \quad$ - Speed $[\mathrm{km} / \mathrm{h}]$,

$K$ - Density [veh/km],

$Q$ - Traffic volume (intensity) $[\mathrm{veh} / \mathrm{h}]$

\footnotetext{
* Marija Kopf ${ }^{1}$, Petr Cenek $^{2}$

${ }^{1}$ Department of Transportation Networks, Faculty of Management Science and Informatics, University of Zilina, Slovakia,

E-mail: mkopf@mkopf.de
} 


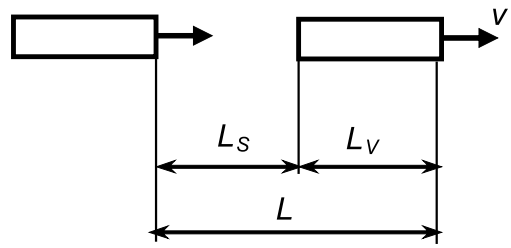

Fig. 1 The Car Following Process

The density stands for an average number of vehicles on a unit road length. It can be estimated as

$$
K=\frac{1}{L}
$$

The traffic volume is defined as an average number of vehicles that pass a certain point on a road during a unit time interval. The following equation gives the basic characteristics of a steady flow

$$
Q=K^{*} v
$$

The calibration of the microscopic systems is a challenging task. The simulation system must be accurate in order to be applicable for solving the transportation problems.

After the calibration parameters are chosen, the simulation model must be validated for its accuracy. The formal representation of how the model corresponds to reality is as follows:

$$
\operatorname{PR}[\mid \text { reality - simulation_prediction } \mid<\delta]>\alpha
$$

The symbol $\delta$ represents the difference between the simulation model and reality, $\alpha$ is the probability that the acceptable difference $\delta$ is met. The metrics suitable for measuring the difference between the reality and the predicted results obtained from the simulation.

The inputs for the simulators are of various forms and origin. Geometric inputs, like lane width, junctions etc, are available through documented map sources. Others are obtained by calibrating the system, i.e. using the field data. The field data can be classified in the following categories.

- Parameters that can be directly estimated from field data (vehicle mix, arrival rates, turning percentages)

- Parameters not directly measurable (drivers aggressiveness)

- Tuning parameters that are not real but are required for the model (free flow speed, lost time)

The traffic states can be determined by the statistical analysis of traffic flow data observed from surveillance videos at two freeway locations in the network. The vehicle characteristics and performance data included vehicle length, maximum speed, maximum acceleration and deceleration rates, etc.

\section{Vision Surveillance Framework}

This chapter explains the framework of such a system and shows the layer where this work aims to search for the error sources.

Nearly every visual surveillance system starts with motion detection. Motion detection aims at segmenting regions corresponding to moving objects from the rest of an image. Subsequent processes such as tracking and behavior recognition are greatly dependent on it. The process of motion detection usually involves environment modeling, motion segmentation, and object classification, which intersect each other during processing.

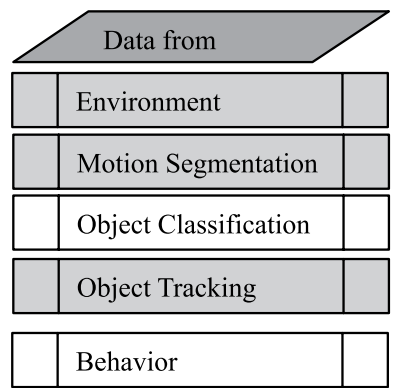

Camera calibration and projection geometry Vehicle position estimation

Vehicle velocity and acceleration estimation

Vehicle velocity and acceleration estimation

Fig. 2 Vision Surveillance Framework

After the motion detection, surveillance systems generally track moving objects from one frame to another in an image sequence. The tracking algorithms usually have considerable intersection with motion detection during processing. Tracking over time typically involves matching objects in consecutive frames using features such as points, lines or blobs. Tracking methods are divided into four major categories: region-based tracking, active-contour-based tracking, feature based tracking, and model-based tracking.

Behavior understanding involves the analysis and recognition of motion patterns, and the production of high-level description of actions and interactions.

\section{Position and Velocity Estimation}

The described framework shows the sequence of steps used for the traffic surveillance. For each step there is a possibility to choose between different methods, depending on the application of interest. Each chosen method in each framework step is an error source. For estimating traffic states in this work, the following steps depicted in Fig. 2 will be considered:

- Data from Camera

- Environment Modeling

- Motion Segmentation

- Object Tracking

Possible errors when collecting data from camera are related to the camera calibration and projection geometry. Errors due to environment modeling relate to the precise estimation of the 

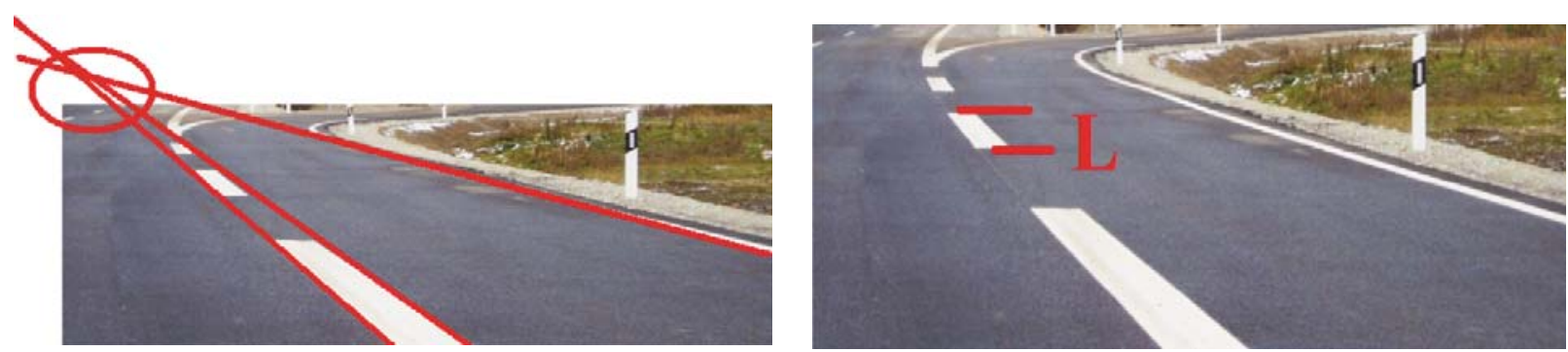

Fig. 3 Position Estimation: Determining the vanishing point and using landmarks

vehicle position. Possible errors during motion segmentation and object tracking are related to the estimation of velocity and acceleration. The problems to solve are how to estimate the real dimensions from a video picture, depending on the camera position and the road position in the video image. It is possible to use the GPS to measure the position of chosen landmarks. The accuracy of GPS measurements of absolute positions is in the range of $0.01 \mathrm{~m}$ to $15 \mathrm{~m}$. The theoretical analysis is done to predict the precision and errors which can be expected for each of the parameters.

The second error source is the quantization both in time and in space. The traffic states are derived from the sequence of video images. The position of the vehicle is evaluated in each video frame. These values give information about the velocity and acceleration. The video sequence is discrete in time, since it consists of individual frames, and in space, since the image consists of individual pixels. It will be evaluated what filtering can be used and the influence of the sampling frequency on the accuracy of results.

The frequency of frames is an important parameter for the calculation of velocity and acceleration. The influence of the frequency on the velocity and acceleration error will be evaluated. It is obvious, that the higher velocities alse demand the higher frequency of the video frames. It will be considered what minimum frame frequency is necessary for evaluation of vehicle velocity and acceleration depending on the range of these traffic states. The theoretical analysis will be done to determine, depending on the measured velocity, what frequency rates are sufficient so that the increase of frame frequency above this value results in no significant decrease in error. In case, that the vehicles overlap, there is a problem of identifying the seperate vehicles. A vehicle either remains unidentified, or it is not identified in certain frames. This problem can be significantly reduced with the increase of the frame frequency, since the vehicles, if not identified in all frames, would be identified in some of them and by carefully choosing the algorithms for vehicle tracking.

\section{Calibration of the Microscopic Systems}

The statistical data obtained from the surveillance systems are used as input of the model generator of a microscopic simulation system. How to obtain the vehicle count, velocity and acceleration was described in previous chapters. The velocity of an observed vehicle is measured at the specific points in the scene to enable modeling of the driver behavior.
The figure below shows one measurement of successive velocities at the junction to model the behavior of drivers when turning to the right.

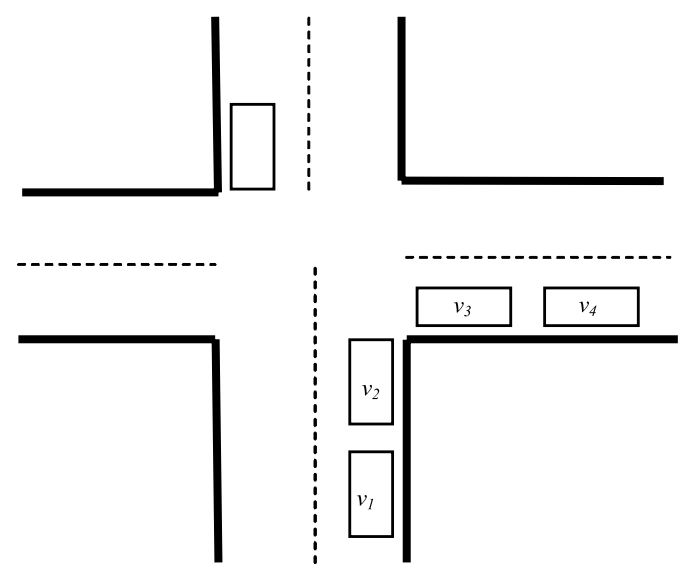

Fig. 4 Velocity estimation at various positions in the scene

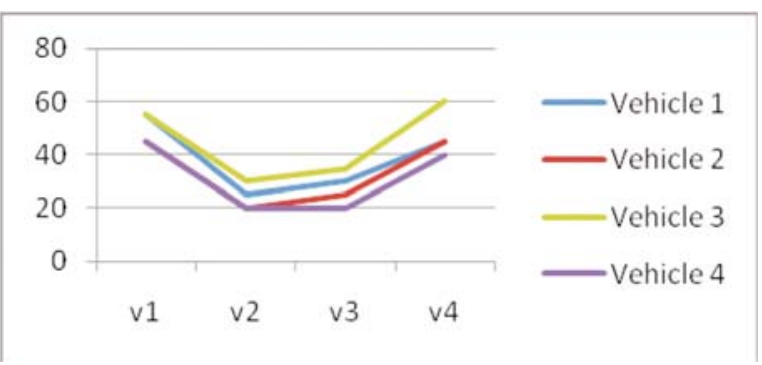

Fig. 5 Velocity profiles of various driver behaviors

\section{Conclusion}

The further work will concentrate on the implementation steps. The implementation of the image processing methods as a software program should enable practical validation of the proposed method improvements. The video data collected with cameras will be processed. The calculated traffic states are going to be used as the calibration parameters of the simulated model. The simulation model at the University of Zilina will be used. The model shall be calibrated with the gained traffic states describing the driver behav- 
ior. The simulation will deliver the simulated traffic sequences which can be used to verify the results. Based on these results, the final evaluation of results and conclusion will be made. The statistical evaluation of the results will be also performed.

\section{References}

[1] CENEK, P.: Models and Optimization in Transports and Logistics. University of Zilina, 2008

[2] PURSULA, M.: Simulation of Traffic Systems - An Overview. Journal of Geographic Information and Decision Analysis, vol.3, 1/1999, pp.1-8

[3] BAYARRI, B.: Assessing Uncertainties in Traffic Simulation: A Key Component in Model Calibration and Validation. TRB 83rd Annual Meeting, pp. 32-40, January 2004

[4] SEUL, M.: Practical Algorithms for Image Analysis. Cambridge University Press, 2000, ISBN-10: 052188411X

[5] JIMINEZ, T.: A Road Traffic Simulator: Car-Following and Lane- Changing. Proceedings of the $14^{\text {th }}$ European Simulation Multiconference on Simulation and Modelling, 2000

[6] KUMAR, P.: Framework for Real-Time Behavior Interpretation From Traffic Video. IEEE Transactions on Intelligent Transportation Systems, Vol. 6, 1/2005

[7] GRAMMATIKOPOUlOS, L., KARRAS, G., PETSA, E.: Automatic Estimation of Vehicle Speed from Uncalibrated Video Sequences, International Symposium on Modern Technologies, Education and Professional Practice in Geodesy and Related Fields, Sofia 2005

[8] ZHANG, Z.X. , LI, M., HUANG, K.Q., TAN, T. N.: Robust automated ground plane rectification based on moving vehicles for traffic scene surveillance, $15^{\text {th }}$ IEEE International Conference on Image Processing, 2008

[9] BOSE, B., GRIMSON, E.: Ground Plane Rectification by Tracking Moving Objects, Proc. of Joint IEEE International Workshop on Visual Surveillance and Performance Evaluation of Tracking and Surveillance, 2003

[10] H. RAKHA, B. HELLINGA, M. VAN AERDE, W. PEREZ: Systematic Verification, Validation and Calibration of Traffic Simulation Models, Annual Meeting, TRB, Washington, DC, 1996

[11] MASOUD, O., ROGERS, S., PAPANIKOLOPOULOS, N.: Monitoring Weaving Sections, Report no. CTS 01-06, University of Minnesota, USA, October 2001

[12] GONZALES, R., R. WOODS, R., EDDINS, S.: Digital Image Processing, $2^{\text {nd }}$ Edition, Prentice Hall; 2nd edition (January 15, 2002), ISBN-10: 0201180758

[13] SEUL, M. et al.: Practical Algorithms for Image Analysis, Cambridge University Press, 2000, ISBN-10: 0521660653. 